\title{
Implementation of Welding Defects Detection and Monitoring in Arc Welding Robots
}

\author{
Gsimalseed Ali Gsimalseed Gsimalla ${ }^{1}$, Jiang Dening ${ }^{2}$ \\ ${ }^{1,2}$ Tianjin University of Technology and Education (TUTE), School of Electronics Engineering Tianjin, China
}

\begin{abstract}
The objective of this paper is to develop and add new features to ABB arc welding robots using image processing, supervisory control and data acquisition (SCADA)systems. aback propagation artificial neural network (ANN) model was designed for distinguish welding defects and SCADA system fixed and mobile SCADA also was designed using Arduino shield module. Although only the color indices associated with image pixels were used as inputs, it was assumed that the ANNs model could develop the ability to use other information's, such as shapes and implicit in these data. The images were taken in the field and then cropped to depicting only the welding beads, either good quality of welding bead or poor welding. The data base of the images has different types of good quality of weld beads and poor weld beads. The ability of the ANNs to discriminate good weld beads from poor weld bead was tested on $30 \%$ of the images. The others have been used for training purposes. For ANNs, the success rate for classifying good weld bead is the highest as 90\%.where as the highest success rate for poor weld bead recognition is $80 \%$. This is considered satisfactory for the given limit amount of training data and the computer hardware limitations. Monitoring of the welding process data and control it by Arduino shield help users to tracking and control the process easily.
\end{abstract}

Keywords: Artificial neural networks (ANNs), welding beads, Roi, SCADA, ABB arc welding robots, Arduino shield

\section{Introduction}

Robotics automation systems are rapidly taking on the place of the human work force. One of the benefits is that this change provides the human work force with the time to spend on more creative tasks. Industry has benefited drastically from the expanse of a robotic work force. Automated machines have taken over the duties of dangerous and mundane jobs from humans, allowing greater productivity. Because robots never tire, extra shifts have been added to factories. During welding process there is welding defects happens and this defects make mechanical weakness in these parts which can put human being life in danger [15] specially for ship and loaded parts of the cars.

It was decided to use ANNs in this study to Specify, the possibility of using ANNs to distinguish between images of arc welding process (good weld and poor weld) in case of poor weld take the part out of production line and reprocess it and monitoring that at data monitoring systems .Images captured in real-time by a digital camera, and use microcontroller to control arc weld machine directly from $\mathrm{ABB}$ arc welding robot controller in case of welding defects detected. In this paper the use of ANNs was confined to the differentiation three welding defects commonly happened in manufacture arc welding process.

The most common welding defects were power defects (undercurrent, over current), robots speed defects and gun defects. Fig (1) show over view of welding defects detection and monitoring system

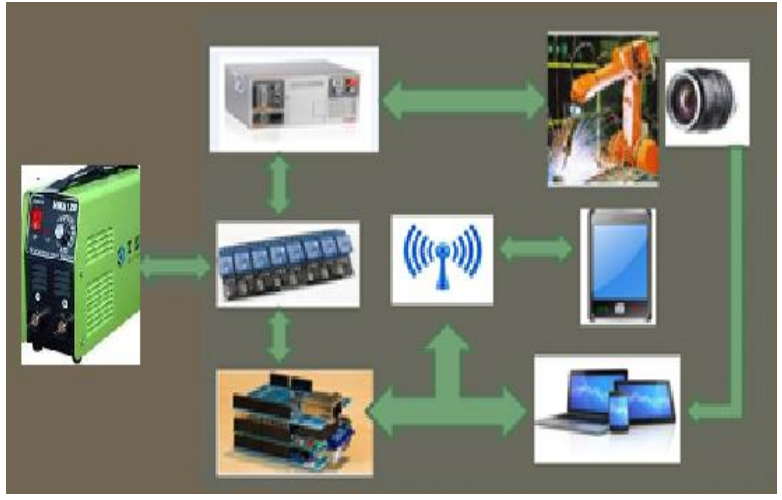

Figure 1: welding defects detection and monitoring system.

\section{Welding Defects}

Welding defects in this paper classified to ampere defects, travel speed defects and gun defects.

Setting the ampere too low will be result in a tall narrow bead lacking in penetration. The weld will be difficult to start and the arc prone to straying towards one side of a joint in preference to the other.

When Amps too high The bead is wide, flat and irregular, and a small undercut can be seen on the right of the weld in the fig. 2 a deep crater has formed at the end of the weld.

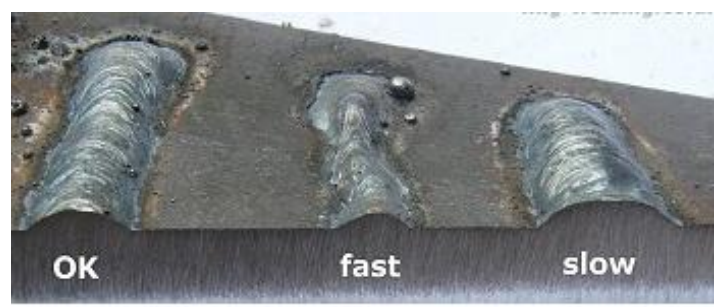

Figure 2: Current Setting defects

In addition to that travel speed weld defects it will be like when speed too fast excessive speed results in a thin, weak 


\section{International Journal of Science and Research (IJSR) \\ ISSN (Online): 2319-7064}

Index Copernicus Value (2013): 6.14 | Impact Factor (2014): 5.611

bead. The ridges in the weld are elongated and triangular. When Speed too slow welding too slowly results in a wide tall build up of weld. [9]

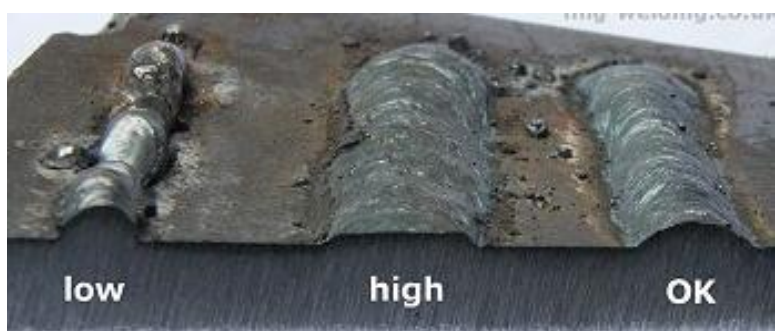

Figure 3: Travel speed defects

For arc length defects arc length too short this weld was laid with the end of the rod covered by the molten slag. Arc length too long too great a distance between the rod and the work will increase the voltage resulting in a flat and wide weld with a great deal of spatter.

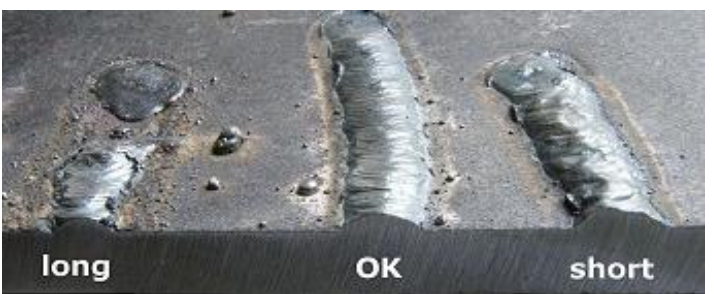

Figure 4: Arc length defects

\section{Embedded Systems Design}

Intermediate relay designed to couple Arduino Uno MCU and Arduno shield module with the arc weld robot and also to control welding machine.

All this embedded system works as server for web SCADA .In addition to that Arduino Uno \& shield works as server for visual studio to design Pc SCADA in the same time visual studio works as a Server for the mat lab to get the image processing result and transmit it to the Ardunio. Fig(5) show the general overview of the systems.

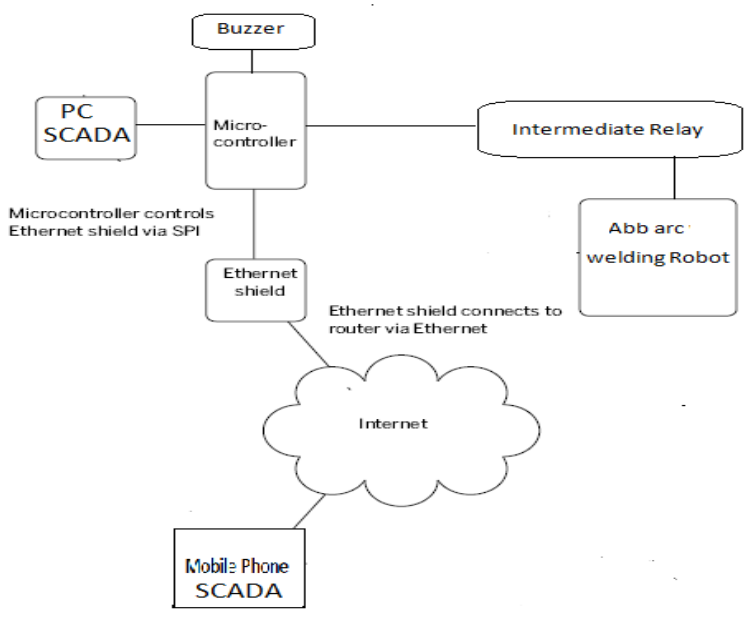

Figure 5: general overview of the system

\subsection{Hardware system}

1) Microcontroller AVR atmega328 (ArduinoUno).
2) Arduino Ethernet shield.

3) Intermediate relay.

4) Digital camera.

5) Light adjust plate.

6) Buzzer.

\subsubsection{Microcontroller}

The Arduino Uno is a microcontroller board that uses the ATmega328 microcontroller. The board itself comes with everything needed to support the microcontroller. Fig (6)show arduino uno

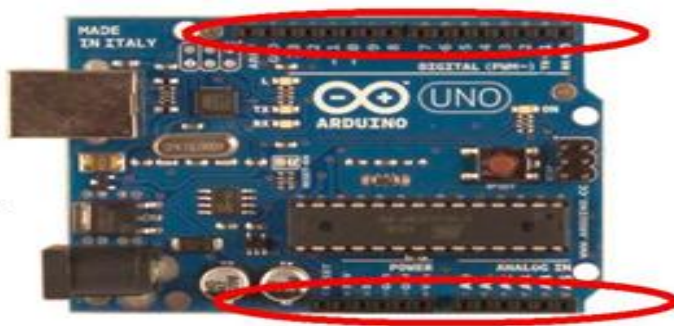

Figure 6: arduino uno

\subsubsection{Arduino Ethernet shield}

The Arduino Ethernet shield allows the Arduino board to connect to the internet. It uses the Wiznet W5100 Ethernet chip which provides a network stack for the Arduino. Fig (7) show Arduino Ethernet shield.[15]

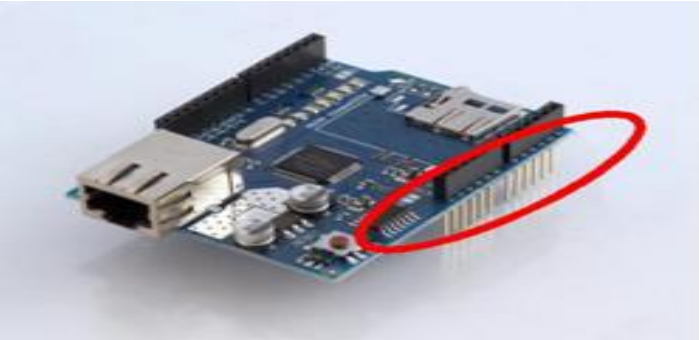

Figure 7: Arduino Ethernet shield

\subsubsection{Intermediate Relay}

Intermediate relay works as interfacing tool between $\mathrm{ABB}$ robot controller and the $\mathrm{PC}$ according to the voltage level difference arduino Uno works as driver for this relay fig $(8)$ show the circuit and it contain TIL111 optical coupler to isolate the difference voltage level safely. Also fig (9) show Intermediate relay connected to the PC and controller using arduino board

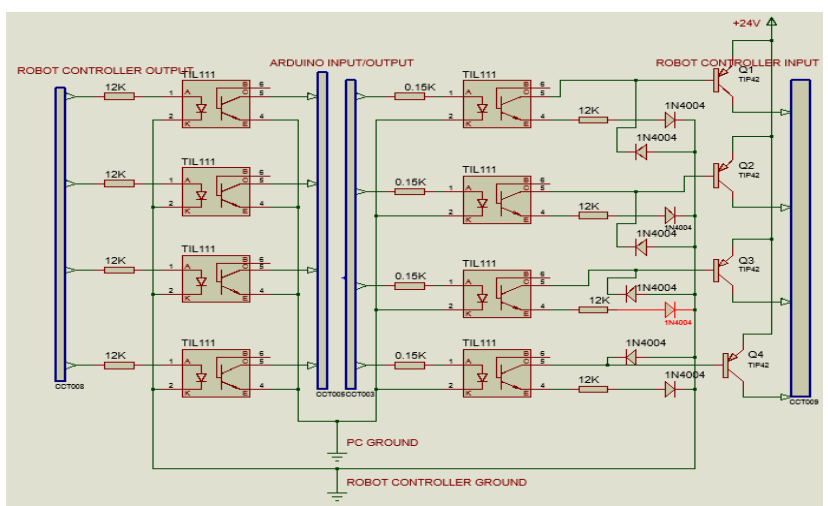

Figure 8: Intermediate Relay circuit 


\section{International Journal of Science and Research (IJSR) \\ ISSN (Online): 2319-7064}

Index Copernicus Value (2013): 6.14 | Impact Factor (2014): 5.611

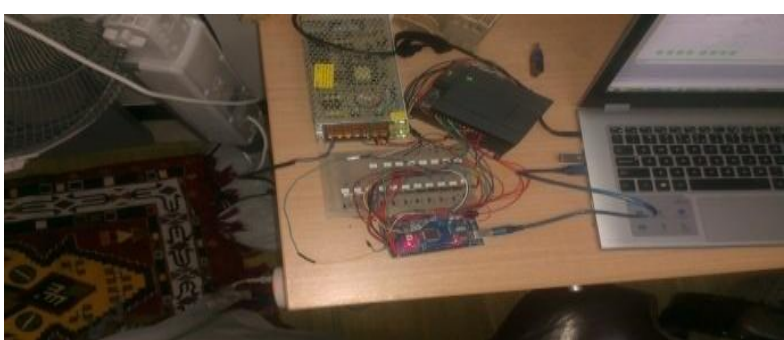

Figure 8: Testing Intermediate Relay

\subsubsection{Digital camera (Logitech c920)}

In this research Logitech c920 digital camera used and it records in full1080p at 30 frames per second resolution $3 \mathrm{Mp}$ native sensor onboard video compression (H.264) [14].

\subsubsection{Light adjust plate}

Visible light from welding processes is very bright and can overwhelm the ability of the camera to take the image the function of the plate is to limit the brightness of the light reaching the camera. The result is that camera can capture a good quality images.

\section{1) The software}

The software's were programmed used to build the systems of that are described below.

- Robot Studio allows robot programming to be done on a PC.

- Arduino for connection ( robot with scada and robot with web).

- Visual studio for the PC scada and works with matlab as server for image processing for the robot.

- HTML for web scada designing.

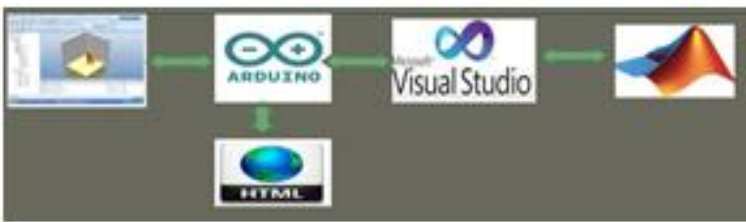

Figure 10: software map.

\section{2) System and web Server}

Arduino board is considered as the heart of the system along with an Ethernet Shield utilized for web server implementation. The Arduino program interacts with the visual basic and Visual basic interacts with matlab software code when the Ethernet Shield hosts the server along with GUI designs. The Ethernet Shield is also fitted with a memory card for hosting the GUI programs.

A connection diagram between the Arduino board and the Ethernet Shield is provided in Fig. (11). There are four connections for SPI and two other connections for enabling the Ethernet control and memory card. In this project a micro memory card is plugged in on the Ethernet shield, which is holding an index.htm file along with the GUI code written in html and Java script.

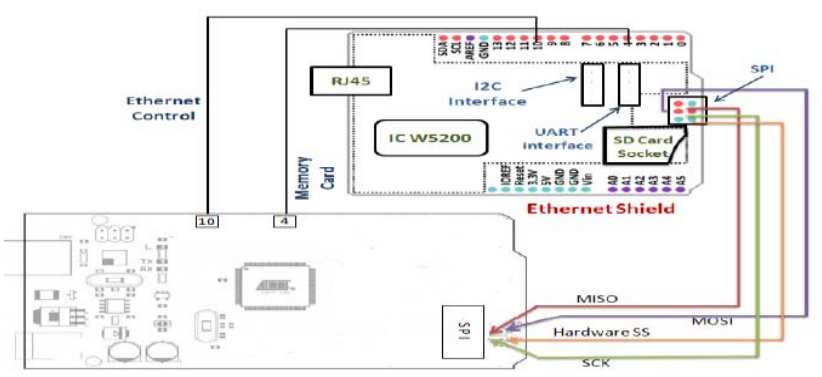

Figure 11: connection diagram between the Arduino board and the Ethernet Shield.

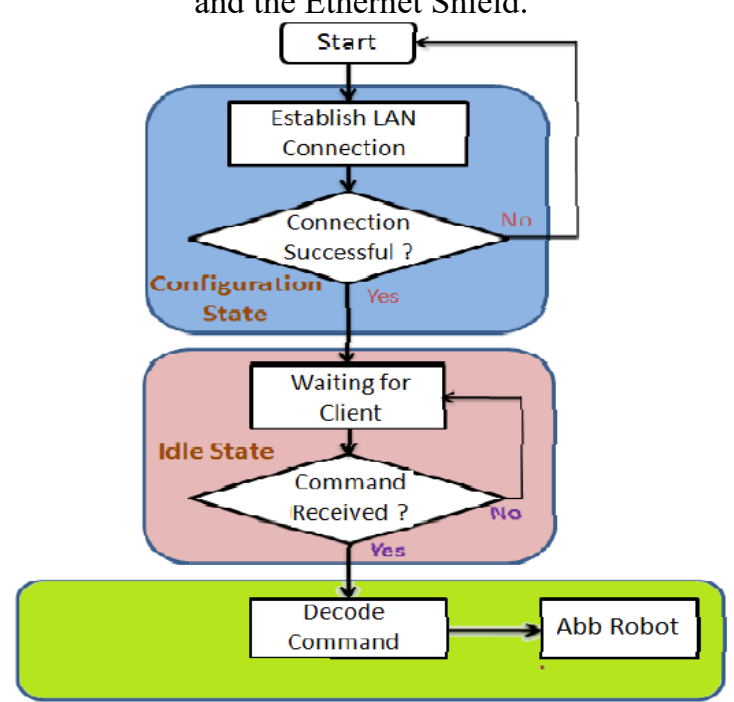

Figure 12: Flow chart for connection establishment with internet.

Fig.(12) show the flowchart of three major tasks of this program configuration state, idle state and robot control is shown in Fig (12). When the client makes a request or any other action happens within the client's browser, it sends a request using http. When the server identifies and accepts the request protocol, it then generates and sends an http response to the client's browser. Once a connection between a client and server has established, it will then enter into the configuration stage. Here the port number and url of the server are checked to establish the connection to the server.

If the connection is successful, then the server waits for a command. The server enters the idle mode until it receives any command. When the server receives a command, it will then allow users to take control of the robot and welding machine.

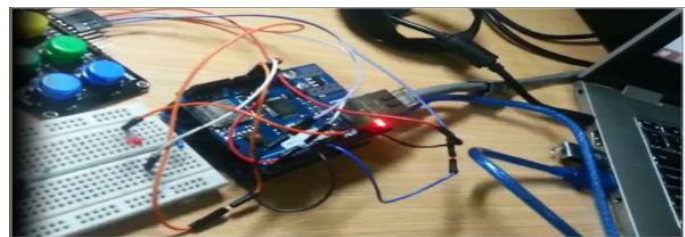

Figure 13: Robot web server testing 


\section{International Journal of Science and Research (IJSR) \\ ISSN (Online): 2319-7064}

Index Copernicus Value (2013): 6.14 | Impact Factor (2014): 5.611

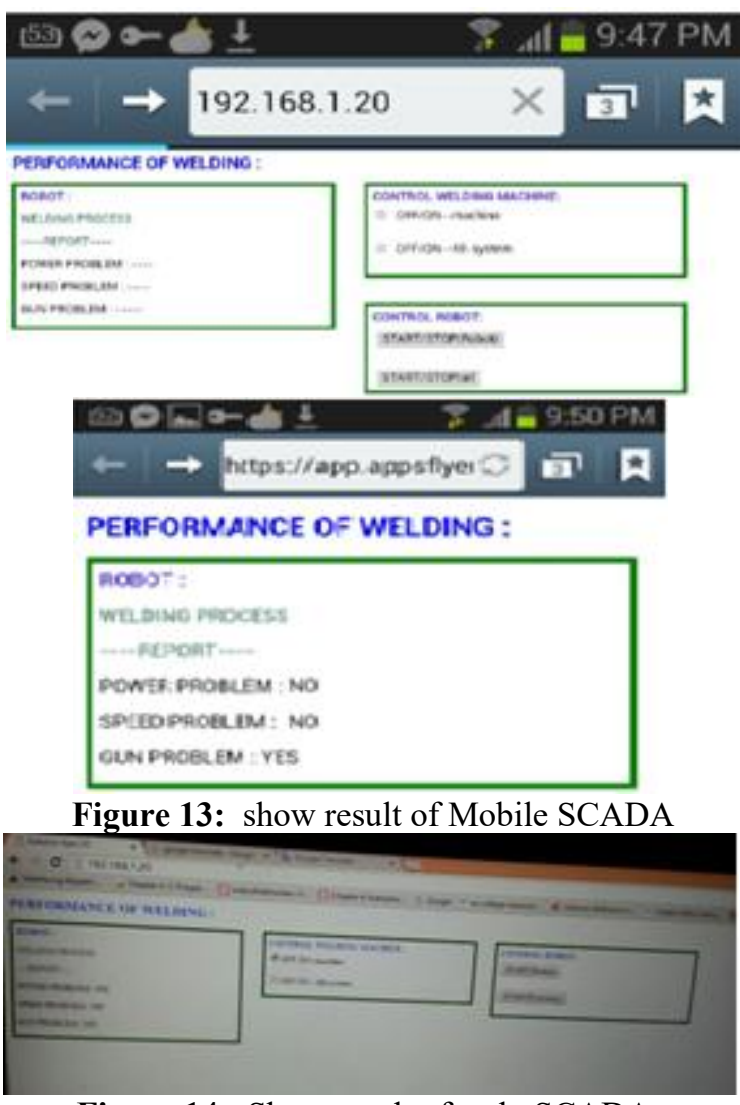

Figure 14: Show result of web SCADA.

\section{Overview of Algorithm}

In this research the processing algorithm can be divided into three parts: Pre-processing stage -involves steps such as image acquisition, definition of a region of interest (ROI),Processing stage which involve processing techniques such as Filtering, Segmentation, Normalization and Enhancing the final result and Post-processing stage- in which process like features extraction and classification are involved. Overall block diagram for welding image processing algorithm is shown in the fig.(16) below.

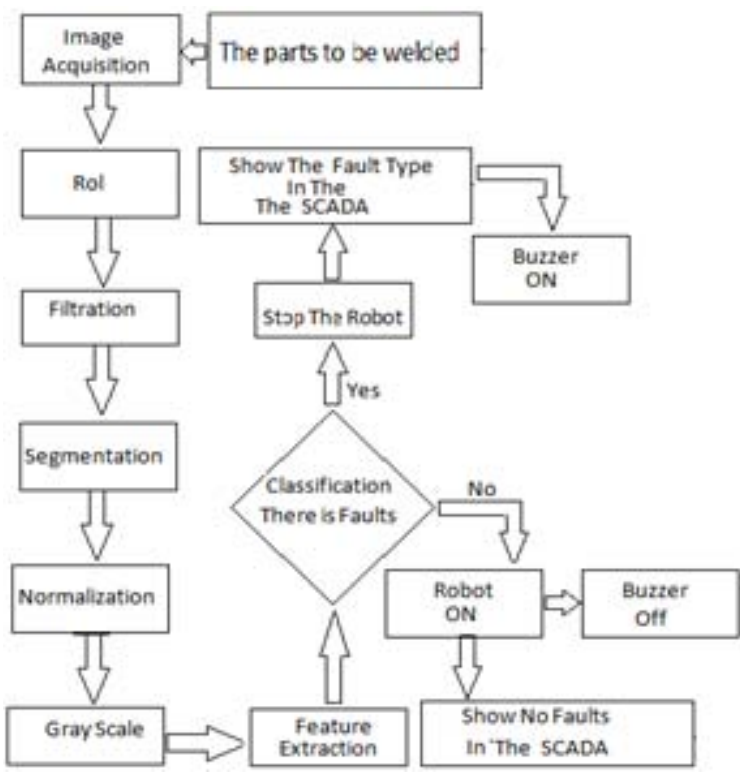

Figure 16: overall block diagram for welding image processing algorithm
After the images were obtained and preprocessed with the Image Processing toolbox for Mat lab (Math Works 2013a). The images were converted to indexed images based on a red-green-blue (RGB) color system. Each pixel of an image was classified into one of 256 categories, represented by an integer in the range from 0 (black) to 255 (white). Each assigned color index number served as an ANN input . Although the color indices were the only inputs used in this study, other features, such as shapes, were expected to be taken into account by the ANNs since information about them is implicit in the relationships between the pixel colors. The Neural Network toolbox for Mat lab (Math Works 2013a) was used to build the ANN models code.

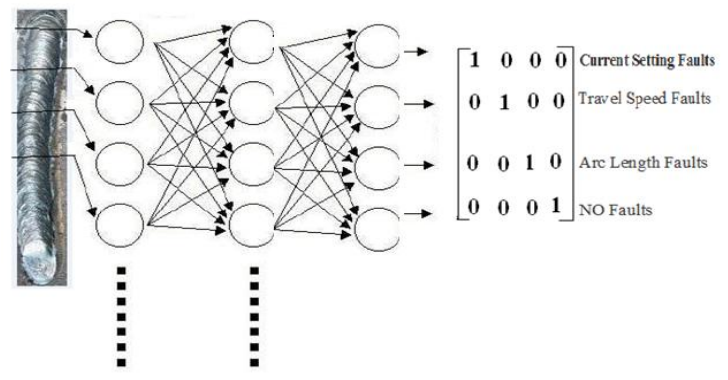

Figure 17: The ANN structure

As shown in Fig (17), there were four output variables; the first was an estimate of the possibility that the object was a current setting defects, and the second was travel speed defects and the third for the possibility of arc weld length defects and fourth for the possibility of good welding process. The data base of the images have different types of good quality of weld beads and poor weld beads.

The ability of the ANNs to discriminate good weld beads from poor weld beads were tested on $30 \%$ of images. The others have been used for this training purposes. The objectives are to address the problem of uncertain classification four images were tested for the ANN module. These are named ANN-A ANN-D. The conditions under which an object was recognized as a good welding quality or a poor welding the Brier scores was calculated. A log sigmoid transfer function was implemented in each PE. During the training procedure of the ANNs, the maximum acceptable sum-squared error was empirically set at $1 \times 10^{-5}$. The training process was carried out with fast back propagation, until the minimum acceptable sum-squared error was reached. A trial-and-error method was used to set the number of PEs in the hidden layer. The success classification rate for each object (good weld or poor weld) was determined after training in order to evaluate the ANN model performance. 


\section{International Journal of Science and Research (IJSR) \\ ISSN (Online): 2319-7064}

Index Copernicus Value (2013): 6.14 | Impact Factor (2014): 5.611

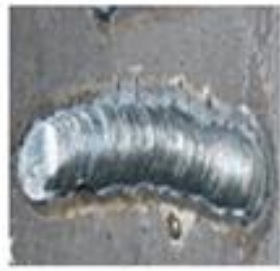

A

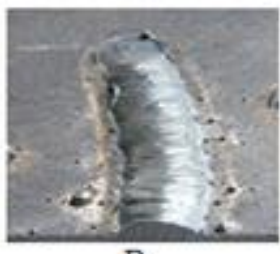

B

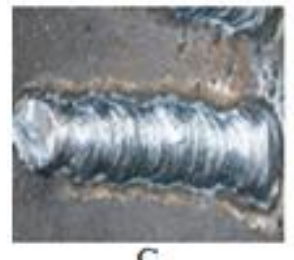

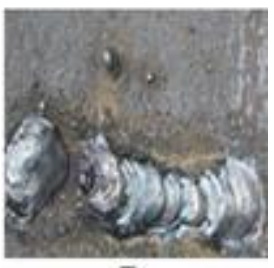

D

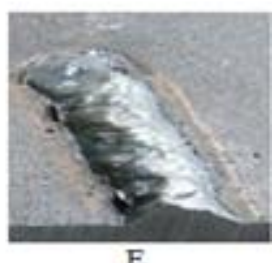

$\mathrm{E}$

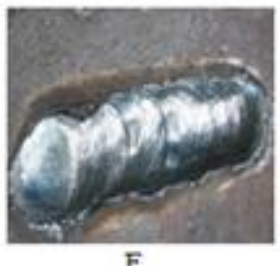

Figure 18: Examples of welding images. (A) and (B) for good welding in training data set; $(\mathrm{C})$ for good welding in test data set; (D) and (E) for poor welding in training data set; and $(\mathrm{F})$ for poor welding in test data set.

\section{Results and Discussion}

The ability for the classification of images using the ANNs and the four target outputs can be evaluated using Brier scores . The range of Brier scores is from 0.239 to 0.358 . Although this model is among the best for welding recognition, the success classification of the ANN is from $60 \%$ to $90 \%$ for good welding beads and from $50 \%$ to $80 \%$ for poor welding beads. Generally low Brier scores indicate the potential capability of ANNs to classify and recognize images.

It is necessary to further analyses the ANNs model performance in detail and carefully consider the success recognition rates for classifying good weld beads and poor weld beads .The Brier scores results indicates that ANNs have ability to classify and distinguish images of good weld beads from images of poor weld beads. In the end using scada we can identify.

- Welding Defects

- Control Robots.

- Control Welding Machines.

\section{Conclusion}

This paper is mainly focused on detecting of the most common welding defects were power defects (undercurrent, over current), robot speed defects , arc length defect iand image processing and Supervisory control and data acquisition (SCADA) systems to detect the common welding bead defects. Therefore it is concluded that an ABB arc welding robots based- ANNs and SCADA system can potentially be used to increase the welding efficiency and observes of the welding process.

\section{References}

[1] Math Works. 2013a. MATLAB Image Processing Toolbox User's Guide.

[2] Math Works. 2013a. MATLAB Neural Network Toolbox User's Guide.

[3] Math Works. Using MATLAB. TheMath Works, Inc.

[4] Programming and interfacing ATMEL's AVRS Jul 29, 2015 by Thomas Grace .

[5] AVR Microcontroller and Embedded Systems: Using Assembly and C (Pearson Custom Electronics Technology) Jan 31, 2010 by Muhammad Ali Mazidi and Sarmad Naimi .

[6] Artificial Intelligence - A Modern approach - Slaurt Russel and Peter Norving, Pearson Education, 2nd Edition

[7] Introduction to Robotics - P.J.Mc Kerrow, Addisson Wesley, USA, 1991 Bernard Sklar, Digital Communications: Fundamentals and Applications, Prentice Hall, 2001.

[8] http://www.csbescgab.ca/docs/journal/42/42_3_147_ocr .pdf.

[9] http://www.mig-welding.co.uk/arc-welding-faults.htm.

[10] L.Perlovsky, "Conundrum of combinatorial complexity,"IEEE Tranactions on Pattern Analysis and MachineIntelligence. Vol.20, no.6, pp.666-670, June 1998.

[11] S.Horikawa, T. Furuhashi, and Y. Uchikawa,"On FuzzyModeling Using Neural Networkswith the BackPropagation Algorithm,'IEEE Trans. on Neural Networks.Vol.3, no.5, pp.801-806, September 1992.

[12] Making Things Talk, 2nd Editionby Tom IgoePublished by O'Reilly Media, Inc.and Classifier Performance in Computer Aided Diagnosis:the Effect of Finite Sample Size," Med Phys.Vol.27, no.1,pp.1509-1522,June2000.

[13] http://www.lth.se/fileadmin/maskinkonstruktion/robotte knik/arkiv/2002/bolmsjo_ind_rob2002.pdf.

[14] http://www.logitech.com/assets/48317/2/webcam-c920c-setup-guide.pdf.

[15]http://www.emeraldinsight.com/doi/pdfplus. 